\title{
Many-body dynamics of the decay of excitons of different charges in a quantum dot
}

\author{
J. A. Andrade, A. A. Aligia, and Pablo S. Cornaglia \\ Centro Atómico Bariloche and Instituto Balseiro, \\ Comisión Nacional de Energía Atómica, CONICET, 8400 Bariloche, Argentina
}

(Dated: February 22, 2018)

\begin{abstract}
We calculate the photoluminescence spectrum of a single semiconductor quantum dot strongly coupled to a continuum as a function of light frequency, gate voltage, and magnetic field. The spectrum is dominated by the recombination of several excitonic states which can be considered as quantum quenchs in which the many-body nature of the system is suddenly changed between initial and final states. This is associated with an Anderson orthogonality catastrophe with a power-law singularity at the threshold. We explain the main features observed experimentally in the region of stability of the trion $X^{-}$, the neutral exciton $X^{0}$ and the gate voltage induced transition between them.

PACS numbers: 78.67.Hc, 75.20.Hr, 78.60.-b
\end{abstract}

The optical manipulation of semiconductor quantum dots (QDs) is a subject of great interest because of its potential use to control the electronic spin for quantum information processing [1-3] and spintronics [4 6 ]. Different optical means of manipulation [6] and detection [5] of the the spin have been proposed. The core of the research in this area consists of optical transitions involving either neutral excitons or trions. These states are respectively bound states of one or two electrons in the conduction band of the QD and a hole in the valence band, and can be tuned using a gate voltage $V_{g}[1,3,7+11]$.

An ubiquitous aspect of the photoluminescence (PL) decay of excitons of various charges $9-13$, or the absorption of light creating them [13 15, is the manifestation of the hybridization of the orbital of the QD with a continuum of extended states. Small QD systems can usually be described by variations of the Anderson impurity model. For small hybridization and an odd number of electrons in the QD, this model reduces to the Kondo model, in which the localized spins have an exchange interaction with the spins of the electrons in the reservoir [9] resulting in a many-body singlet ground state [16.

The PL spectrum that results from the decay of the trion $X^{-}$[10, 11] and the neutral exciton $X^{0}$ [11] has been measured as a function of $V_{g}$. The $X^{-}$PL line is a consequence of an optical transition to a state with a single electron on average in the QD. As a consequence of the hybridization, the PL is broad near the limits of stability of the trion, it is asymmetric and there is a nonmonotonic blue shift. While simple approaches were able to explain these features [10, 11, 17] a fully reliable calculation is still lacking. For the $X^{0}$ decay, similar nontrivial effects are present 11 . The creation of the $X^{0}$ [15] and $X^{-}[14$ states by optical absorption were calculated using the numerical renormalization group (NRG), and in the $X^{-}$case shows a remarkable agreement with experiment.

These transitions, because of their sudden character, are related to another field of great interest in recent years, the dynamics of highly correlated systems, after a quantum quench [14, 15, 18, 22. Because initial states (IS's) and final states (FS's) have different local scattering potentials, the spectrum should show at low temperatures a power-law behavior at the PL threshold characteristic of $\mathrm{x}$-ray edge singularities [13, 15, 23. This is due to the Anderson orthogonality catastrophe [24, which is another cornerstone of many-body physics, and requires sophisticated techniques for its treatment.

An important aspect of the experiment of Kleemans et al. 11] is that they worked in the regime of strong hybridization and studied the transition between the $X^{0}$ and $X^{-}$decays. In this Letter, we calculate the PL on the whole range of gate voltages $V_{g}$ between the region of stability of $X^{-}$and $X^{0}$, using NRG within the fulldensity matrix approach [25 29]. Our results provide an explanation of recent experiments and show that a very different behavior of the PL spectrum under an applied magnetic field is expected for the $X^{0}$ and $X^{-}$decays.

Using Fermi's golden rule, the PL intensity is given by

$$
I(\omega)=\frac{2 \pi}{\hbar} \sum_{i f} w_{i}\left|\left\langle f\left|H_{L M}\right| i\right\rangle\right|^{2} \delta\left(\hbar \omega+E_{f}-E_{i}\right),
$$

where $\omega$ is the PL frequency, $|i\rangle$ labels the IS, $|f\rangle$ denotes the possible FS's, $E_{j}$ is the energy of the state $|j\rangle, w_{i}$ is the Boltzmann weight of the IS $|i\rangle$ and the relevant part of light-matter interaction can be written as 17.

$$
H_{L M}=A\left(d_{\uparrow}^{\dagger} p_{3 / 2}+d_{\downarrow}^{\dagger} p_{-3 / 2}\right)+\text { H.c. },
$$

where $d_{\sigma}^{\dagger}$ creates an electron at the QD with spin $\sigma$, and $p_{m}$ annihilates a valence electron with angular momentum $3 / 2$ and projection $m$ [1, 31. Eq. (1) implies a sudden change in the dynamics of the system equivalent to a quantum quench.

The Hamiltonian is written as 


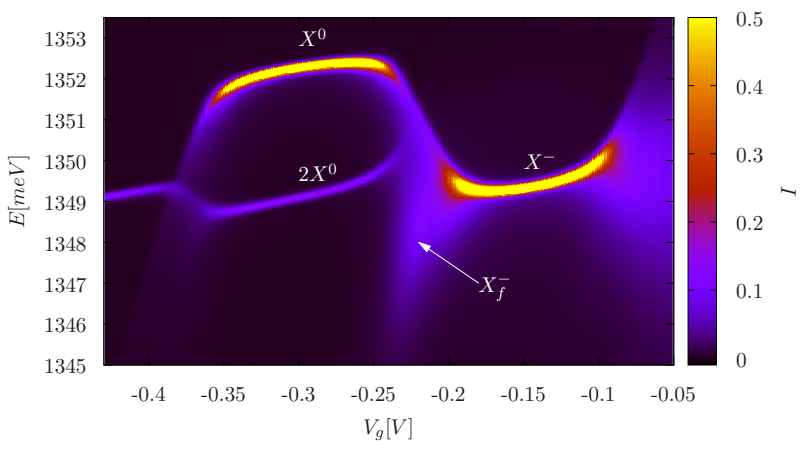

FIG. 1. (Color online) PL spectrum as a function of gate voltage. The intensity is normalized by twice the maximum intensity at $V_{g}=-0.35 \mathrm{~V}$ 31. Parameters are $T=1.75 \mathrm{~K}$, $E_{e}^{0}=-25 \mathrm{meV}, E_{h}^{0}=1398.5 \mathrm{meV}, U_{h h}=U_{e h}=U_{e e}+2 U_{e e}^{\prime}=$ $20.97 \mathrm{meV}, U_{e e}^{\prime}=3.52 \mathrm{meV}, \Delta=1 \mathrm{meV}$, and $\lambda=8$.

$$
\begin{aligned}
H= & E_{e} n_{d}+U_{e e} n_{d \uparrow} n_{d \downarrow}+\sum_{k \sigma}\left(V_{k} d_{\sigma}^{\dagger} c_{k \sigma}+\text { H.c. }\right) \\
& +\sum_{k \sigma} \epsilon_{k} c_{k \sigma}^{\dagger} c_{k \sigma}+E_{h} n_{h}+U_{h h} n_{h 3 / 2} n_{h-3 / 2} \\
& -U_{e h} n_{d} n_{h}+U_{e e}^{\prime} n_{d \uparrow} n_{d \downarrow} n_{h} .
\end{aligned}
$$

Here $h_{m}^{\dagger} \equiv p_{m}, n_{d \sigma}=d_{\sigma}^{\dagger} d_{\sigma}, n_{h m}=h_{m}^{\dagger} h_{m}, n_{d}=$ $n_{d \uparrow}+n_{d \downarrow}$, and $n_{h}=n_{h 3 / 2}+n_{h-3 / 2}$. The first three terms correspond to the well known Anderson impurity model. The remaining terms involve heavy holes, The last term takes into account the increase of the repulsion between electrons as their wave function contracts after addition of holes. Its addition improves the agreement with experiment simultaneously for the range of $V_{g}$ of both, the $X^{-}$and the biexciton $2 X^{0}$ decay. Contrary to previous approaches, we consider the hybridization in both IS's and FS's. The on-site energies of the QD electrons and holes change with gate voltage as $E_{e}=E_{e}^{0}-e V_{g} / \lambda$, and $E_{h}=E_{h}^{0}+e V_{g} / \lambda$, respectively, where $\lambda$ is the lever arm [10, 11$]$

Some parameters can be determined by simple features of the experiment [10, 11]. For example, neglecting $V_{k}$, the amplitude of range of voltage in which the PL spectrum is dominated by the decay of the trion $X^{-}$is $V_{e e}^{1}=$ $\lambda U_{e e} / e$ (see Fig. 2). We obtain a good agreement with experiment for the parameters indicated in Fig. 11. We assumed the hybridization $\Delta=\pi \sum_{k}\left|V_{k}\right|^{2} \delta\left(\epsilon_{F}-\epsilon_{k}\right)=1$ $\mathrm{meV}$ independent of energy with support in the range $[-D, D]$, associated with a wide reservoir band of width $2 D=100 \Delta$ symmetrically placed around the Fermi energy $\epsilon_{F}$. There are no particular features of the band or hybridization that can affect the results.

We use the NRG to calculate the PL and compare with the experiments of Kleemans et al. [11. We run the code two times, one in the presence of one or two holes in the valence band of the QD (IS) and the other for one hole less (FS). Then, the density matrix for the IS's and the

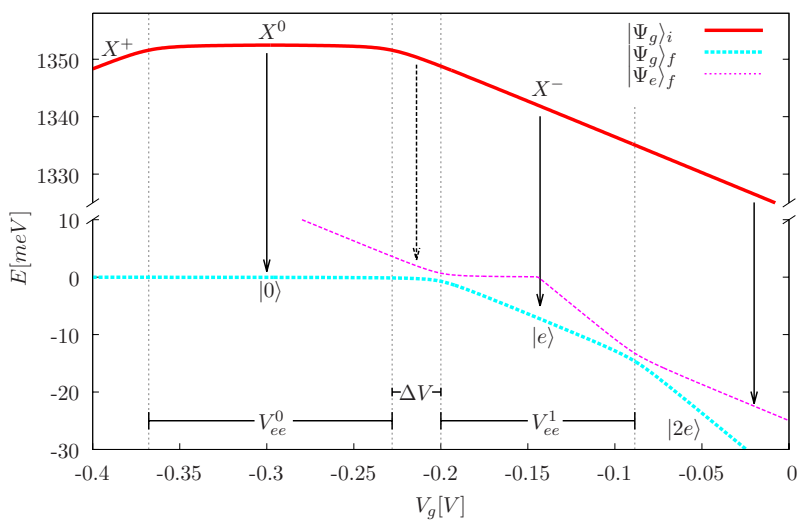

FIG. 2. (Color online) Most relevant energy levels of the zero-bandwidth model and the PL transitions (indicated with arrows). $\left|\Psi_{g}\right\rangle_{i}$ and $\left|\Psi_{g}\right\rangle_{f}$ are the initial and final ground states, respectively. $\left|\Psi_{e}\right\rangle_{f}$ is an excited FS. Here $V_{e e}^{0}=$ $\lambda\left(U_{e e}+U_{e e}^{\prime}\right) / e, V_{e e}^{1}=\lambda U_{e e} / e$ and $\Delta V=\lambda\left(U_{e h}-U_{e e}-U_{e e}^{\prime}\right) / e$. The hopping between the QD level and the reservoir effective level is $V=1 \mathrm{meV}$. Other parameters as in Fig. 1 .

matrix elements entering Eq. (2) are calculated (see e.g. Refs. 23] and [14]). In Fig. 1 we show the resulting PL spectrum as a function of $V_{g}$. There are two high intensity plateaus that correspond to the decay of $X^{0}$ (lower $V_{g}$ ) and $X^{-}$(greater $V_{g}$ ). Near the crossover between them, the plateaus bend and the $X^{-}$plateau is joined by another transition line of lower frequency and intensity, which corresponds to that denoted as $X_{f}^{-}$in Ref. [11. There is another plateau of lower intensity which corresponds to the decay of a biexciton, with two holes and two electrons to the FS $X^{0}$. This feature is denoted by $2 X^{0}$ in the experiment 31. Except for the presence of positively charged Mahan excitons and multiple excitons which involve electron or hole states not included in the Hamiltonian, our results agree with those of Kleemans et al. 11.

While the line shape of the PL peaks (discussed below) requires a sophisticated calculation, the evolution of them with $V_{g}$ and the transition between states of different total charge, can be understood qualitatively using a molecular model in which the band width is reduced to a single electron reservoir state with an energy equal to $\epsilon_{F}(D \rightarrow 0)$ 32]. The most relevant states of this model with less than two holes are represented in Fig. 2. The initial ground state (IGS), before light emission, depending on the value of $V_{g}$ consists mainly of either a single hole $\left(X^{+}\right)$in the valence band of the $\mathrm{QD}$, or $X^{0}$ or $X^{-}$, joined by regions with some admixture between $X^{+}$and $X^{0}$ or $X^{0}$ and $X^{-}$due to the hybridization. The FS's after light emission have no hole in the valence band and, similarly to the IS's, mainly zero $(|0\rangle)$, one $(|e\rangle)$ or two electrons $(|2 e\rangle)$ in the QD. The arrows in Fig. 2 correspond to the main transition in each voltage regime. The feature denoted as $X_{f}^{-}$corresponds to the transition, indicated by a dashed arrow, from the 


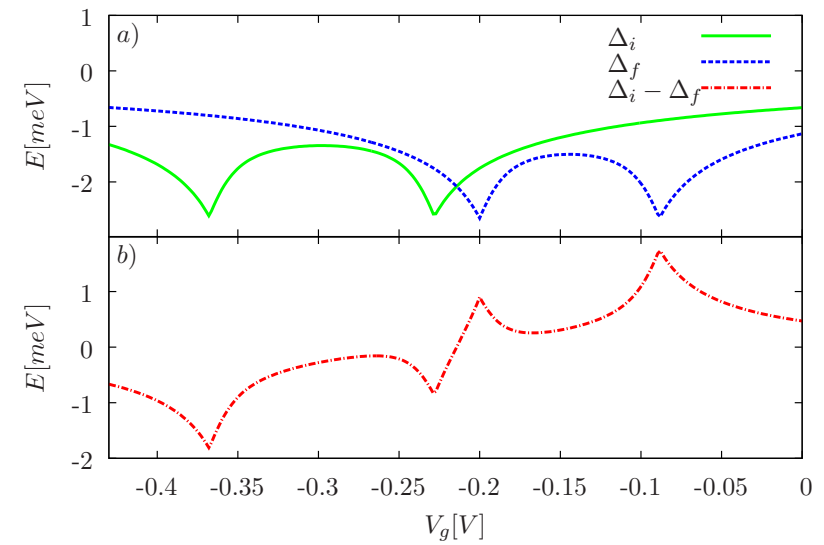

FIG. 3. (Color online) a) Green full line: energy gain $\Delta_{i}$ of the IS before photoemission due to hybridization. Blue dashed line: energy gain $\Delta_{f}$ for the FS after photoemission. b) Change of the emitted photon energy gain $\Delta_{i}-\Delta_{f}$ due to hybridization.

trion to an excited state with a dot occupancy between zero and one. The observed plateaus in the PL spectrum can be understood in the $V=0$ limit. The transition $X^{0} \rightarrow|0\rangle$ takes place, with an emission energy $\hbar \omega=E_{e}^{0}+E_{h}^{0}-U_{e h}$, in the interval $E_{e}^{0}-U_{e h} \leq e V_{g} / \lambda \leq$ $E_{e}^{0}-U_{e h}+U_{e e}+U_{e e}^{\prime}$. The high $V_{g}$ plateau in Fig. 1. with an energy $E_{e}^{0}+E_{h}^{0}-2 U_{e h}+U_{e e}+U_{e e}^{\prime}$, stems from the electron-hole recombination of the trion $X^{-} \rightarrow|e\rangle$, in the range $E_{e}^{0} \leq e V_{g} / \lambda \leq E_{e}^{0}+U_{e e}$.

When the hybridization is turned on, both the IS and FS energies decrease by $\sim \Delta$. These energy gains calculated with NRG-FDM and their difference, which gives the shift in PL frequency, are represented in Fig. 3. As expected, the energy gain is larger near the intermediate valence regions. For example, there is a dip in the energy of the FS at $V_{g}=\lambda E_{e}^{0} / e=-0.2 \mathrm{~V}$ for which the occupancy of the FS at the dot $n_{d}$ is intermediate between 0 and 1 . Another dip is clear for $V_{g}=\lambda\left(E_{e}^{0}+U_{e e}\right) / e=-0.088 \mathrm{~V}$ for which $n_{d} \approx 1.5$. Note that even in the Kondo regime $V_{g} \approx-0.144 \mathrm{~V}$ for which $n_{d} \approx 1$, the energy gain is of order of $\Delta$ in contrast to expectations from simple approaches [11, 17]. For $V_{g} \geq \lambda E_{e}^{0} / e$, the net effect of the hybridization is a blue shift which is more pronounced for intermediate valence occupancy of the FS's. Similarly, in the region of the $X^{0}$ decay, the effect of the hybridization is a red shift, larger near the limits of stability of $X^{0}$ against either $X^{+}$or $X^{-}$. These shifts explain characteristic features of the PL frequency as a function of gate voltage $V_{g}$ observed in the experiment and reproduced in Fig. 1. In particular the downward curvature in the $X^{0}$ region and an upward curvature in the $X^{-}$region. A similar reasoning can be followed for the $2 X^{0}$ feature leading to the same qualitative behavior as for the $X^{-}$decay, in agreement with the results shown in Fig. 1

In Fig. 4 we show two PL line shapes that correspond

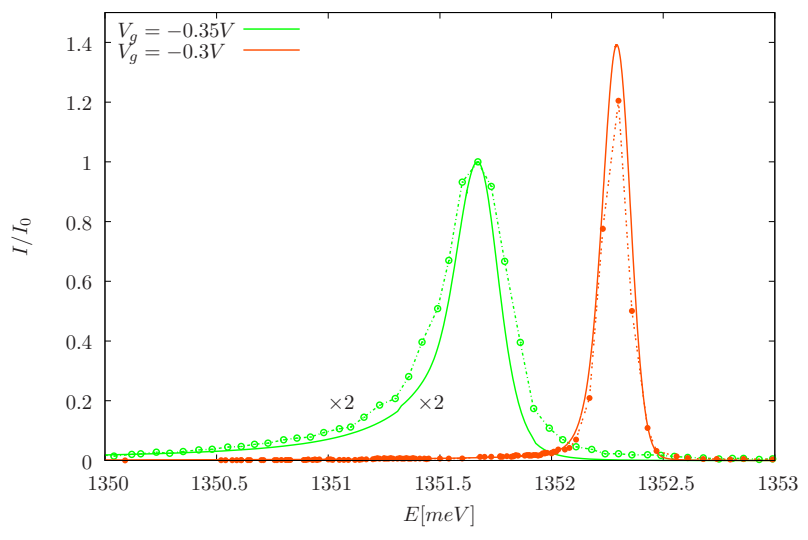

FIG. 4. (Color online) PL intensity close to the onset of the $X^{0}$ plateau (green solid line) and at the center of the $X^{0}$ plateau (orange solid line). The orange filled and green open circles are the corresponding Kleemans digitized results 11. $I_{0}=I(1351.6 \mathrm{meV})$ for $V_{g}=-0.35 \mathrm{~V}$.

to the $X^{0}$ decay for $V_{g}=-0.35 \mathrm{~V}$ and $-0.3 \mathrm{~V}$ and the respective experimental results digitized from Ref. [11. The comparison is excellent. The long tails at low energies provide evidence of the excitations produced by the sudden change of the dynamics of the system (quantum quench) associated with an Anderson orthogonality catastrophe. To discuss this point in more detail, in Fig. 5 we show the PL intensity shift from the threshold $\omega_{e}^{*}$ in a logarithmic scale, for temperatures much smaller than the Kondo temperature $T_{K}$ and three values of the gate voltage: $V_{g}=-0.144 \mathrm{~V}$ corresponding to the Kondo regime for the FS in the $X^{-}$decay, $V_{g}=-0.214 V$ in the region between the $X^{0}$ and $X^{-}$decays, where the PL frequency changes strongly as a function of gate voltage and both IS's and FS's are in the intermediate-valence regime, and lastly for $V_{g}=-0.3 \mathrm{~V}$ corresponding to the Kondo regime for the IS in the $X^{0}$ decay. In the first case, as the frequency is lowered, we recover the same regimes studied theoretically before for absorption of light creating $X^{0}$ [15]. At high frequency, the physics is dominated by the free-orbital fixed point of the NRG, with a dependence $I(\omega) \sim \omega^{2}$. Lowering the frequency the functional form turns to that of the local moment fixed point until the Kondo frequency $k_{B} T_{K} / \hbar$ is reached. For $\hbar \omega<k_{B} T_{K}$, the physics enters the strong-coupling regime, and the dependence is the characteristic power law $\omega^{-\eta_{\sigma}}$ for the Anderson orthogonality catastrophe associated with the quantum quench in a Fermi liquid. The exponent is given by [15] $\eta_{\sigma}=1-\sum_{\sigma^{\prime}}\left(\delta_{\sigma \sigma^{\prime}}-\Delta n_{\sigma^{\prime}}\right)^{2}$, where $\Delta n_{\sigma^{\prime}}$ is the change on the local occupation of electrons with spin projection $\sigma^{\prime}$, between the FS and IS. This divergent behavior at low frequency ceases at a frequency $k_{B} T / \hbar$ determined by the temperature. For $V_{g}=-0.214 \mathrm{~V}$, the behavior is similar, except for the absence of the local moment regime and the different exponent $\eta_{\sigma}$. For $V_{g}=-0.3 \mathrm{~V}$ ( $X^{0}$ decay $)$, the local moment regime can- 


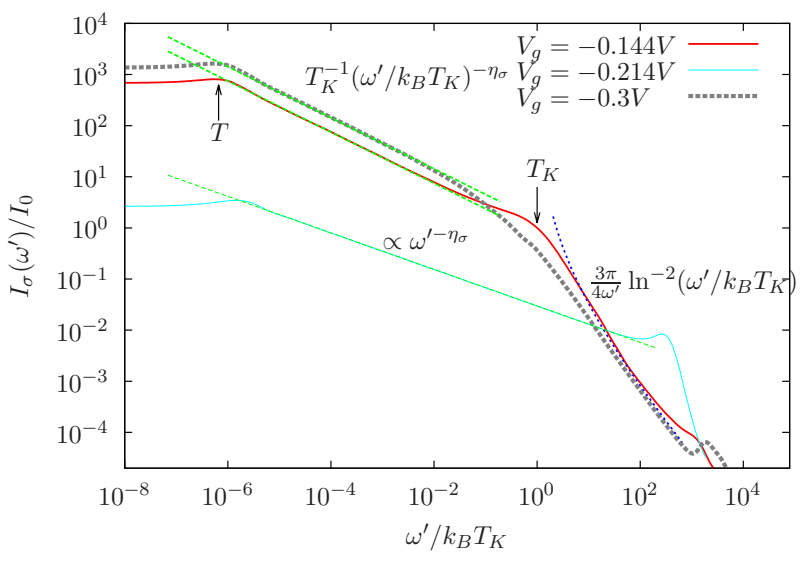

FIG. 5. (Color online) PL intensity as a function of the frequency $\omega^{\prime}=\omega_{e}^{*}-\omega$ shift from the threshold $\left(\omega_{e}^{*}\right)$ in a $\log -\log$ scale for three values of the gate voltage, $-0.144 \mathrm{~V}$, $-0.214 \mathrm{~V}$ and $0.3 \mathrm{~V}$. The threshold are $1349.74 \mathrm{meV}, 1350.34$ $\mathrm{meV}$ and $1352.54 \mathrm{meV}$, respectively. The intensity is normalized by $I_{0}=I\left(k_{B} T_{K} / \hbar\right)$ calculated for $V_{g}=-0.144 V$, where $T_{K} \sim 0.083 K$.

not be reached and the PL intensity is about four times smaller at $\omega \sim k_{B} T_{K} / \hbar$ than for the $X^{-}$decay. The result is a smoother curve, similar to light absorption leading to $X^{-}[14$.

From the occupancies of the different states, we obtain $\eta_{\sigma}=0.4987$ for $V_{g}=-0.144 V, \eta_{\sigma}=0.3571$ for $V_{g}=-0.214 \mathrm{~V}$ and $\eta_{\sigma}=0.4983$ for $V_{g}=-0.3 \mathrm{~V}$ The corresponding curves $\omega^{-\eta_{\sigma}}$ are also represented in Fig. 5 with the intensity as the only fitting parameter.

Finally, we analyze the effect of an external magnetic field $B[?]$. As it can be seen in Fig. 6, $B$ leads to a splitting of the PL plateaus due to the different giromagnetic factors for electrons and holes [14]. The different broadening of the PL peaks is due to the dependence on $B$ of $\eta_{\sigma}$ (see Fig. 7) [15]. In the regime of the $X^{0}$ decay, the low energy plateau has a much lower intensity and is rapidly suppressed with increasing $B$. This is due to the reduced probability of finding an electron with a high energy spin projection in the IGS. For the $X^{-}$decay however, the IGS has enough energy available to decay to a state with a large occupation of the high energy spin projection and both peaks have a large intensity.

In summary, using an Anderson impurity model, we can explain experimental results of PL in a wide range of gate voltages, including those for which either IS's or FS's or both are in the mixed valence regime. The PL transition is an experimental realization of a quantum quench associated with another realization of Anderson orthogonality catastrophe, but in contrast to previous studies, in our system electron correlations are important in both, IS's and FS's states in the photon decay. We find a marked asymmetry in the PL line shapes for the neutral exciton and trion decays both in the hybridization induced energy shift and on the dependence with mag-

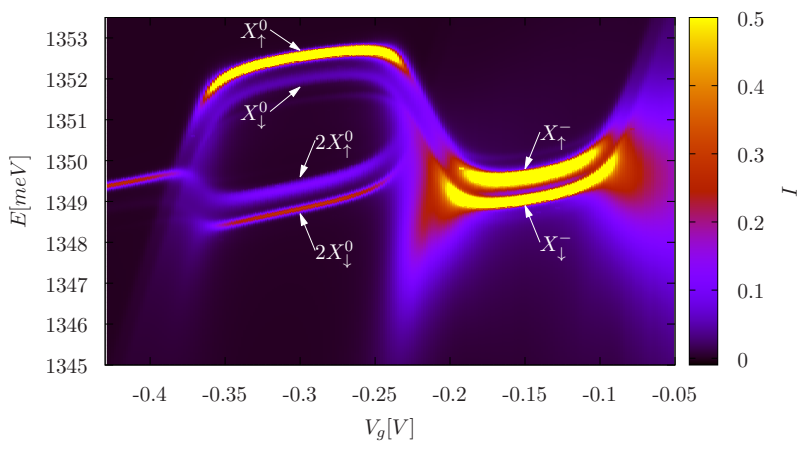

FIG. 6. (Color online) PL intensity in the presence of an external magnetic field producing a Zeeman splitting $0.57 \mathrm{meV}$ of the electronic level of the QD and $\mathrm{a} \sim 1.05 \mathrm{meV}$ splitting for the hole level at $T=1.75 \mathrm{~K}$.

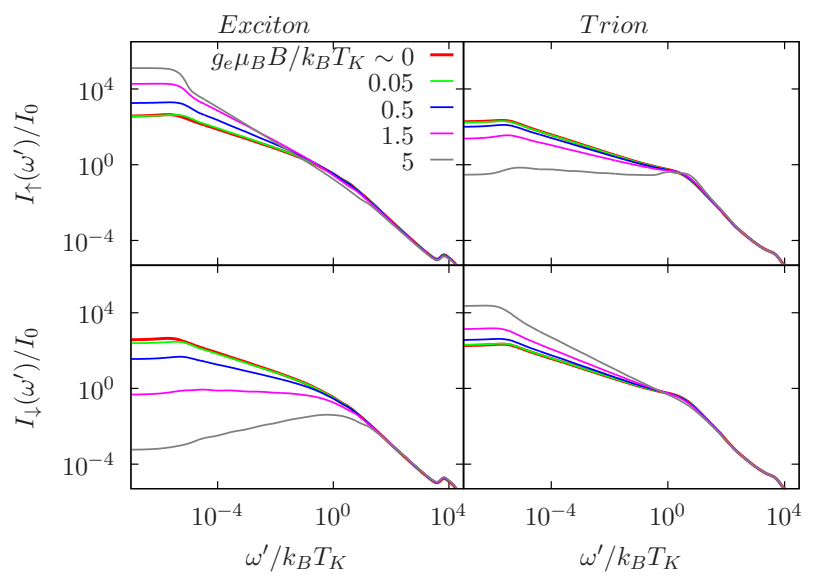

FIG. 7. (Color online) PL intensity as a function of the frequency shift from the threshold for different values of the magnetic field. The left side panels are in the exciton decay regime $\left(V_{g}=-0.3 \mathrm{~V}\right)$ and the right side panels are for trion decay $\left(V_{g}=-0.144 V\right)$. Other parameters as in Fig. 5 .

netic field. Absorption and emission of light are qualitatively different and the local moment regime can be reached only in the FS ( $X^{0}$ absorption or $X^{-}$decay).

We are supported by CONICET. This work was sponsored by PIP 112-201101-00832 of CONICET and PICT 2013-1045 of the ANPCyT.

[1] M. Atatüre, J. Dreiser, A. Badolato, A. Högele, K. Karrai, and A. Imamoglu, Science 312, 551 (2006).

[2] A. Greilich,R. Oulton, E. A. Zhukov, I. A. Yugova, D. R. Yakovlev, M. Bayer, A. Shabaev, Al. L. Efros, I. A. Merkulov, V. Stavarache, D. Reuter, and A. Wieck, Phys. Rev. Lett. 96, 227401 (2006).

[3] J. Berezovsky, M. H. Mikkelsen, N. G. Stoltz, L. A. Coldren, and D. D. Awschalom, Science 320, 349 (2008).

[4] S. Mackowski, T. Gurung, H. E. Jackson, L. M. Smith, G. Karczewski, and J. Kossut, Appl. Phys. Lett. 87, 072502 (2005). 
[5] M. Korkusinski and P. Hawrylak, Phys. Rev. Lett. 101, 027205 (2008).

[6] D. E. Reiter, T. Kuhn, and V. M. Axt, Phys. Rev. Lett. 102, 177403 (2009).

[7] R. J. Warburton, C. Schöflein, D. Haft, F. Bickel, A. Lorke1, K. Karrai, J. M. Garcia, W. Schoenfeld, and P. M. Petroff, Nature 405, 926 (2000).

[8] A. Högele,S. Seidl, M. Kroner, K. Karrai, R. J. Warburton, B. D. Gerardot, and P. M. Petroff, Phys. Rev. Lett. 93, 217401 (2004).

[9] J. M. Smith, P. A. Dalgarno, R. J. Warburton, A. O. Govorov, K. Karrai, B. D. Gerardot, and P. M. Petroff, Phys. Rev. Lett. 94, 197402 (2005).

[10] P. A. Dalgarno, M. Ediger, B. D. Gerardot, J. M. Smith, S. Seidl, M. Kroner, K. Karrai, P. M. Petroff, A. O. Govorov, and R. J. Warburton, Phys. Rev. Lett. 100, 176801 (2008).

[11] N. A. J. M. Kleemans, J. van Bree, A. O. Govorov, J. G. Keizer, G. J. Hamhuis, R. Nötzel, A. Yu. Silov, and P. M. Koenraad, Nature Physics 7, 534 (2010).

[12] S. Cao, J. Tang, Y. Sun, K. Peng, Y. Gao, Y. Zhao, C. Qian, S. Sun, H. Ali, Y. Shao, S. Wu, F. Song, D. A. Williams, W. Sheng, K. Jin, and X. Xu, Nano Research 9, 306 (2016).

[13] R. W. Helmes, M. Sindel, L. Borda, and J. von Delft, Phys. Rev. B 72, 125301 (2005).

[14] C. Latta, F. Haupt, M. Hanl, A. Weichselbaum, M. Claassen, W. Wuester, P. Fallahi, S. Faelt, L. Glazman, J. von Delft, H. E. Türeci, and A. Imamoglu, Nature 474, 627 (2011).

[15] H. E. Türeci, M. Hanl, M. Claassen, A. Weichselbaum, T. Hecht, B. Braunecker, A. Govorov, L. Glazman, A. Imamoglu, and J. von Delft, Phys. Rev. Lett. 106, 107402 (2011).

[16] A. C. Hewson, in The Kondo Problem to Heavy Fermions (Cambridge, University Press, 1993).
[17] L. M. León Hilario and A. A. Aligia, Phys. Rev. Lett. 103, 156802 (2009).

[18] P. Nordlander, M. Pustilnik, Y. Meir, N. S. Wingreen, and D. C. Langreth, Phys. Rev. Lett. 83, 808 (1999).

[19] M. Heyl and S. Kehrein, Phys. Rev. Lett. 108, 190601 (2012).

[20] R. Vasseur, K. Trinh, S. Haas, and H. Saleur, Phys. Rev. Lett. 110, 240601 (2013).

[21] S. L. Lukyanov, H. Saleur, J. L. Jacobsen, and R. Vasseur, Phys. Rev. Lett. 114, 080601 (2015).

[22] A. E. Antipov, Q. Dong, and E, Gull, Phys. Rev. Lett. 116, 036801 (2016).

[23] P. S. Cornaglia and A. Georges, Phys. Rev. B 75, 115112 (2007).

[24] P. W. Anderson, Phys. Rev. Lett. 18, 1049 (1967).

[25] K. G. Wilson, Reviews of Modern Physics 47, 773 (1975).

[26] R. Bulla, T. A. Costi, and T. Pruschke, Reviews of Modern Physics 80, 395 (2008).

[27] M. Yoshida, M. A. Whitaker, and L. N. Oliveira, Phys. Rev. B 41, 9403 (1990).

[28] A. Weichselbaum and J. von Delft, Phys. Rev. Lett. 99, 076402 (2007).

[29] W. C. Oliveira and L. N. Oliveira, Phys. Rev. B 49, 11986 (1994).

[30] In principle, Eq. 2 contains another term in which $d_{\sigma}^{\dagger}$ is replaced by band electrons [11. However this term is small and we have verified that it does not modify substantially our results.

[31] To take into account the smaller probability of excitations of two holes in the experiment, we have multiplied the weight of the initial states with two holes by 0.1 respect to those with one hole.

[32] This molecular model is similar to the zero bandwidth model described in the supplementary information of Ref. 11] but we include all charge configurations. 